\title{
Link adaptation algorithms for improved delivery of delay- and error-sensitive packet-data services over wireless networks
}

\author{
Miguel López-Benítez · Javier Gozálvez
}

Published online: 28 December 2008

(C) Springer Science+Business Media, LLC 2008

\begin{abstract}
Link Adaptation is a radio resource management technique that assesses the channel conditions and selects a transport mode, from a set of possible options, which is optimised for these conditions according to a predefined criterion. The optimum transport mode is commonly determined so as to maximise the throughput. Although this approach may be appropriate for best-effort services, its suitability for multimedia services, usually characterised by tight delay and error performance constraints, has been questioned. As a result, a number of alternative algorithms have been proposed in the literature. In this context, this paper presents and evaluates in a dynamic radio environment several Link Adaptation algorithms designed to enhance the provision of delay- and error-sensitive multimedia packet-data services over wireless systems. The obtained results demonstrate that significant improvements in terms of throughput, transmission delay, error performance and operation of Link Adaptation itself can be obtained with the proposed schemes.
\end{abstract}

Keywords Link adaptation - Radio resource management · Wireless networks - Quality of service provisioning $\cdot$ Multimedia services

\footnotetext{
M. López-Benítez · J. Gozálvez ( $₫)$

Signal Theory and Communications Division, University Miguel Hernández,

Avenida de la Universidad s/n, 03202 Elche, Spain

e-mail: j.gozalvez@umh.es

\section{López-Benítez ( $\square$ )}

Department of Signal Theory and Communications,

Universitat Politècnica de Catalunya, Campus Nord,

C/Jordi Girona 1-3, 08034 Barcelona, Spain

e-mail: miguel.lopez@tsc.upc.edu
}

\section{Introduction}

The introduction of new bandwidth-consuming multimedia services, such as streaming and video conference, the high demand for traditional voice services and the scarcity of available radio resources are creating new challenges to mobile operators that need to implement mechanisms to manage such resources in an efficient and flexible manner. This objective is accomplished by means of Radio Resource Management (RRM) techniques. An important RRM technique with significant benefits and considered in several legacy, recent and future radio technologies is Link Adaptation (LA).

The term LA was originally introduced in the European RACE II ATDMA project [2], which for the first time looked into the practical use of an adaptive coding scheme. This project studied the definition and implementation of an advanced TDMA mobile radio system as a candidate for the Universal Mobile Telecommunications System (UMTS) standard. Thereafter, LA also attracted considerable interest for $2 \mathrm{G}[18]$ and evolved $2 \mathrm{G}$ [12] systems. The radio interface of the Enhanced Data rates for GSM/Global Evolution (EDGE) system was indeed specifically designed for the application of LA [5]. The potential and benefits of LA are such that it has also been considered as a key technique for more recent radio technologies such as High Speed Downlink Packet Access (HSDPA) [16, 17], IEEE 802.11 [19], and IEEE 802.16 [20].

The basis of LA is to assess the channel conditions and then use a transport mode, from a set of possible options, which is optimised for these conditions according to a predefined criterion. A transport mode is defined by a set of parameters affecting the radio transmission operation, for example the coding and/or modulation schemes. Different transport modes are defined to provide varying resilience levels to propagation 
errors under different radio conditions and consequently adapt the transmission data rate. If the experienced radio link quality is favourable then a transport mode with little or no error protection is used. On the other hand, transport modes with extra error protection are selected under poor channel quality conditions. Based on these operating principles, LA periodically adapts the employed transport mode to the experienced varying radio channel conditions. The actual criterion employed to decide the optimum transport mode is a key aspect in the design and performance of LA.

Several criterions to select the optimum transport mode have been proposed in the literature. One of the most accepted and widely used criterions is to select the transport mode that maximises the system throughput based on an off-line process [3]. Although this approach may be appropriate for best-effort services, its suitability for multimedia services, usually characterised by tight delay and error performance constraints, has been questioned. As a result, a number of alternative algorithms have been proposed. As the long-term throughput does not quantify the Quality of Service (QoS) experienced by users of delaysensitive services, the work reported in [15] proposed a LA algorithm designed to reduce transmission delays. To achieve this objective, the proposal in [15] defines a LA algorithm based on the size of the packet to be transmitted. Since certain multimedia services need low packet error rates for not degrading the user perceived QoS, a different approach designed to achieve a given target error rate is proposed in [14]. The work reported in [4] presents an algorithm specifically devised for the provision of video services, aimed at maximising the video quality measured in terms of the Peak Signal-to-Noise Ratio (PSNR). In this context, this paper introduces and evaluates in a dynamic radio environment several LA algorithms designed to enhance the provision of delay- and error-sensitive multimedia packet-data services over wireless systems.

The rest of this paper is organised as follows. First, Sect. 2 describes the LA algorithms presented in [3, 14], and that will be considered as a reference to which compare the performance of the algorithms presented in this paper; such algorithms are described in Sect. 3. Section 4 describes the simulation tool employed to evaluate the performance of the different LA schemes. The obtained results are presented and analysed in Sect. 5. Finally, Sect. 6 summarises the proposals and findings of this research.

\section{Reference link adaptation algorithms}

\subsection{Throughput-based algorithm}

The algorithm described in [3], commonly used in studies involving the use of LA, is aimed at maximising system throughput. The algorithm will hence be referred to as Throughput-based Algorithm (TA). The TA scheme selects the transport mode $T M_{i}$ that maximises the throughput $\Gamma_{T M i}(\gamma)$ under the experienced channel quality $\gamma$. The throughput $\Gamma_{T M i}(\gamma)$ is defined as

$\Gamma_{T M i}(\gamma)=R_{T M i} \cdot\left[1-B L E R_{T M i}(\gamma)\right]$

where $R_{T M i}$ and $B L E R_{T M i}(\gamma)$ represent, respectively, the data-rate and BLock Error Rate (BLER) measured for the experienced channel quality $\gamma$ of the $i$ th transport mode, $T M_{i}$. Therefore, TA decides the optimum transport mode $T M_{\text {opt }}$ for a given channel quality $\gamma$ based on the following procedure

$T M_{\text {opt }}(\gamma)=T M_{x}: \Gamma_{T M x}(\gamma)=\max \left\{\Gamma_{T M i}(\gamma)\right\} \quad \forall T M_{i}$

To illustrate the operation of the TA scheme, let's consider a specific technology such as the General Packet Radio Service (GPRS) system. GPRS offers four different transport modes, each one of them characterised by the use of a different channel-coding scheme as shown in Table 1 . The GPRS transport modes are therefore referred to as Coding Schemes (CSs). These CSs offer a trade-off between throughput and coding protection, paving the way for the application of dynamic LA to GPRS. Since GPRS uses a single modulation scheme, the LA algorithms considered in this paper would only adapt the CS employed. As an example, Fig. 1 shows the throughput performance $\Gamma_{T M i}(\gamma)$ of the GPRS transport modes as a function of the channel quality $\gamma$ expressed in terms of the average Carrierto-Interference Ratio (CIR). As it can be appreciated, the GPRS transport mode offering the maximum throughput depends on the experienced channel quality conditions. In this particular case, CS-1 is considered to be the optimum transport mode by the TA algorithm when the experienced CIR is below $5 \mathrm{~dB} ; \mathrm{CS}-2$ and CS-3 are the optimum transport modes when the CIR is in the range 5-9.5 and 9.5$16.5 \mathrm{~dB}$, respectively, while CS-4 is the optimum transport mode for CIR values higher than $16.5 \mathrm{~dB}$. Theses values determine the LA switching thresholds, which are defined as the boundaries between the regions where each transport mode is regarded as optimum. In more realistic scenarios the performance of transport modes not only depends on the mean channel quality but also on the channel quality distribution among the data block being transmitted.

Table 1 GPRS transport modes

\begin{tabular}{lclc}
\hline Scheme & Code-rate & Payload (bits) & Data rate $(\mathrm{kbit} / \mathrm{s})$ \\
\hline $\mathrm{CS}-1$ & $1 / 2$ & 181 & 9.05 \\
$\mathrm{CS}-2$ & $\approx 2 / 3$ & 268 & 13.4 \\
$\mathrm{CS}-3$ & $\approx 3 / 4$ & 312 & 15.6 \\
$\mathrm{CS}-4$ & 1 & 428 & 21.4 \\
\hline
\end{tabular}


Fig. 1 GPRS transport modes throughput performance versus mean CIR

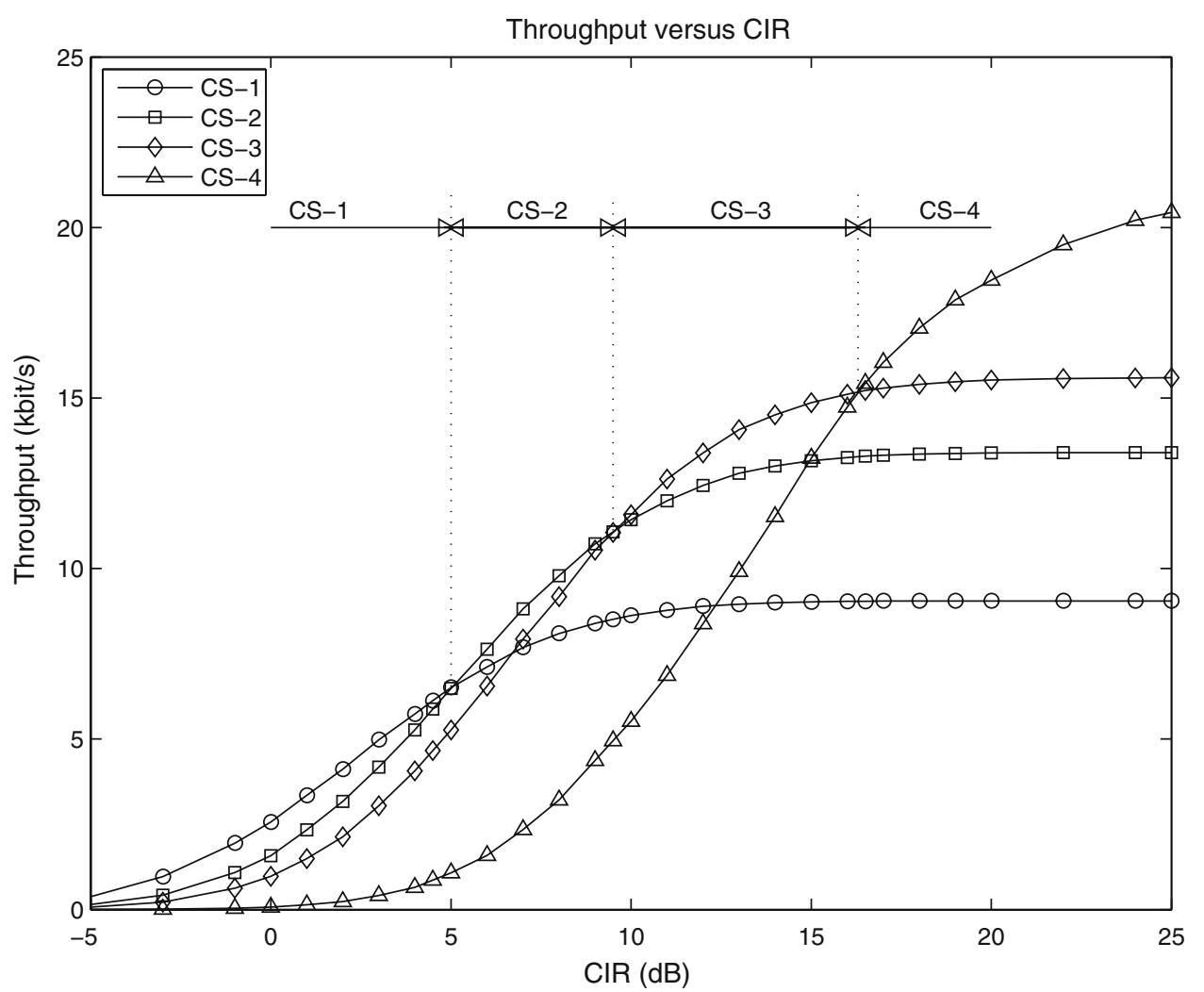

Figure 2 is the analogue to Fig. 1 but considering the throughput performance of the GPRS transport modes as a function of both the mean channel quality, expressed in terms of the experienced mean Bit Error Rate (BER), and the channel quality distribution, represented by the standard deviation of the BER. It is interesting to note from Fig. 2 that the least robust GPRS transport mode (CS-4) is only used when no radio errors occur.

\subsection{Error-based algorithm}

Certain multimedia services are characterised by stringent error rate requirements. The work reported in [14] proposed then a LA scheme aimed at achieving a particular target error rate. The algorithm is therefore referred to as Error-based Algorithm (EA). The switching thresholds for this algorithm are determined using a set of curves relating the error rate to the channel quality. As an example, Fig. 3 illustrates the BLER performance of the GPRS transport modes as a function of the CIR. The LA switching thresholds for the EA algorithm correspond to the channel quality conditions below which a transport mode is not able to further guarantee the target error rate. For instance, assuming a BLER target (BLER $\left.R_{\text {target }}\right)$ equal to 5\%, Fig. 3 shows that the switching thresholds for the EA algorithm correspond to CIR values equal to 13,15 and $24 \mathrm{~dB}$. Note that for CIR values lower than $10 \mathrm{~dB}$ the selected transport mode (CS-1) is not able to provide a BLER lower than the desired $B L E R_{\text {target }}$. However, CS-1 remains the best possible transport mode since it is the most robust alternative. The EA transport mode selection methodology can be expressed as ${ }^{1}$

$$
\begin{aligned}
& T M_{\text {opt }}(\gamma) \\
& =\left\{\begin{array}{ll}
T M_{1}, & B L E R_{T M i}(\gamma)>B L E R_{\text {target }} \quad \forall T M_{i} \\
T M_{x}, & x=\max (i): B L E R_{T M i}(\gamma) \leq B L E R_{\text {target }}
\end{array} \quad \forall T M_{i}\right.
\end{aligned}
$$

As it can be inferred from Fig. 3, when establishing more stringent target error rates (i.e. when reducing $B L E R_{\text {target }}$ ), the EA switching thresholds increase, which promotes a more frequent use of more protected transport modes.

\section{Proposed link adaptation algorithms}

\subsection{Delay-sensitive algorithm}

The first LA algorithm proposed in this work is designed to enhance the provision of delay-sensitive packet-data

\footnotetext{
$\overline{1}$ Expression (3) assumes that transport modes with lower index $i$ values (the minimum value is equal to 1 ) correspond to more robust transport modes.
} 
Fig. 2 GPRS transport modes throughput performance versus mean BER and standard deviation of BER

Fig. 3 GPRS transport modes BLER performance versus mean CIR
Throughput versus mean BER and standard deviation of BER
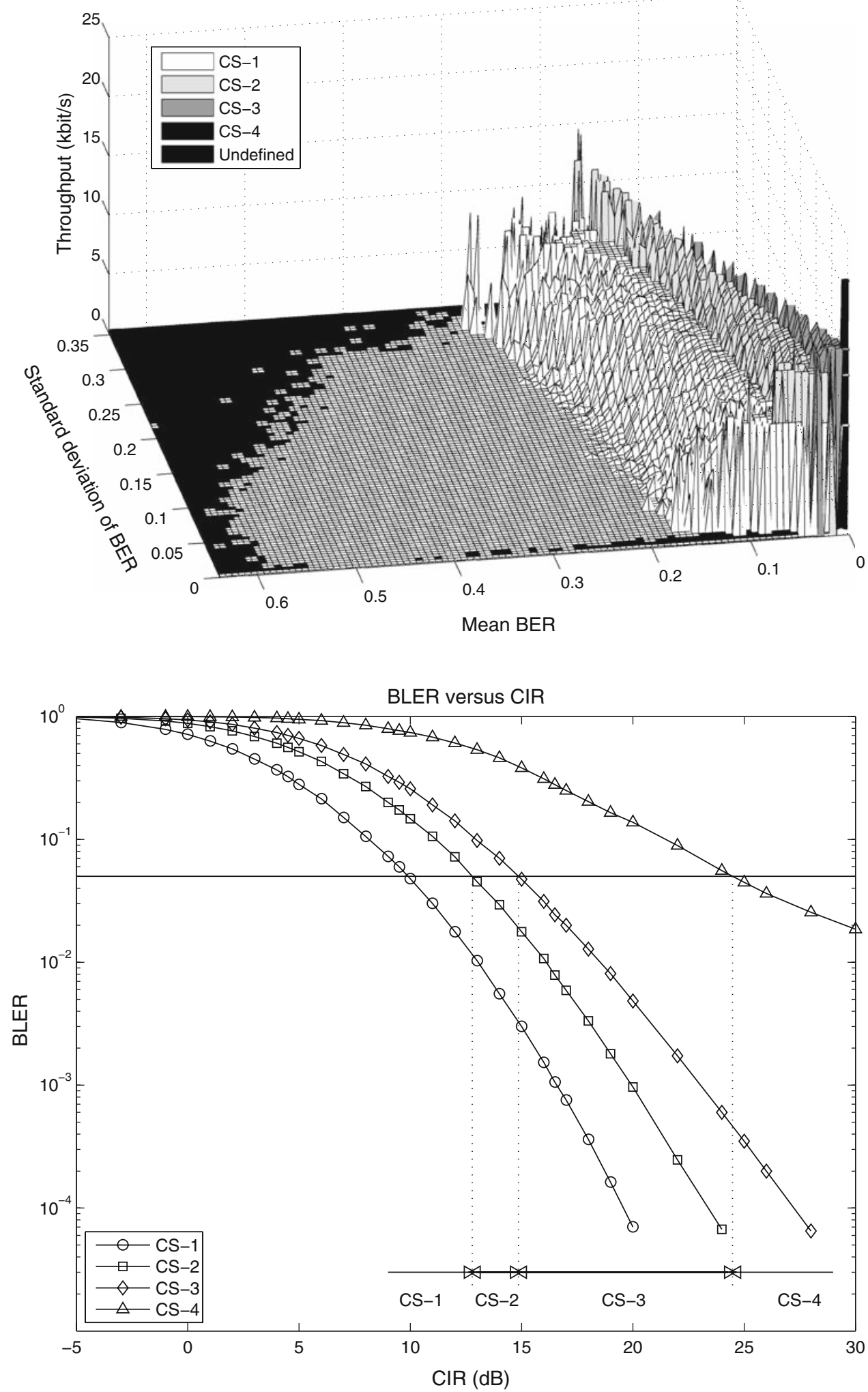

services. The algorithm will hence be referred to as DelaySensitive Algorithm (DSA). Based on the tight delay performance constraints of delay-sensitive traffic, the DSA proposal not only considers the throughput performance as a selection criterion but also the experienced delay, defined as follows. 

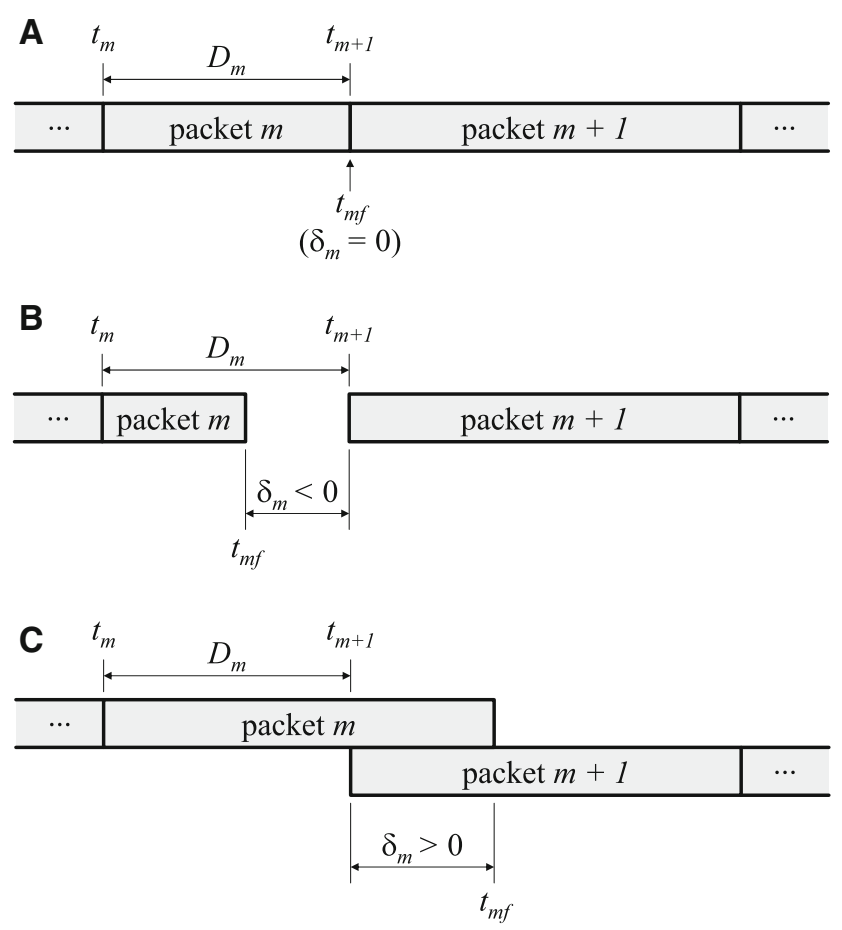

Fig. 4 Packet delay definition

Let's assume that a data-packet $m$ with size $S_{m}$ is generated at time $t_{m}$ and that the next packet is generated at time $t_{m+1}$. We use the term packet to refer to a data block generated at the application layer. For real-time services, the transmission of packet $m$ must be completed in a time interval $D_{m}$ equal to $t_{m+1}-t_{m}$, which will be referred to as packet duration. The time $t_{m f}$ at which the transmission of packet $m$ is actually finished can be expressed as

$t_{m f}=t_{m}+D_{m}+\delta_{m}=t_{m+1}+\delta_{m}$

with $\delta_{m}$ considered as the packet delay. If $\delta_{m}=0$ then $t_{m f}=t_{m+1}$, which implies that the transmission of packet $m$ ends at the same time that packet $m+1$ is generated (see Fig. 4a). When $\delta_{m}<0$ (represented in Fig. 4b), the transmission of packet $m$ finishes before the next packet $m+1$ is generated and the channel can be released to increase the trunking efficiency. On the other hand, if $\delta_{m}>0$ the transmission of packet $m$ has not finished by the time the next packet $m+1$ is generated (see Fig. 4c).

To analytically express the packet delay parameter $\delta_{m}$, we consider that the total time required by a packet to be transmitted over the radio interface depends on the packet size $S_{m}$ and the transmission rate $R_{T M i}$ of the used transport mode. This assumption is reasonable since retransmission protocols, intended to guarantee the integrity of the transmitted data, are frequently deactivated for real-time services in order to not incur in excessive transmission delays (see e.g. [4, 14]). Assuming a fixed transport mode $T M_{i}$ during the packet transmission, the time required to transmit such packet is $t_{m f}-t_{m}=S_{m} / R_{T M i}$. Combining this expression with expression (4) yields

$\delta_{m i}=\frac{S_{m}}{R_{T M i}}-D_{m}$

We can gain some insight into expression (5) by defining $R_{m}=S_{m} / D_{m}$ as the bit-rate required by packet $m$ to be transmitted in a time period equal to $D_{m}$, i.e. with $\delta_{m}=0$. The parameter $R_{m}$ therefore represents the minimum bit-rate required by packet $m$ to be transmitted before packet $m+1$ is generated. Note that if $R_{T M i}<R_{m}$, then $\delta_{m i}>0$ and the transmission of packet $m$ has not finished by the time packet $m+1$ is generated; this case implies a degradation of the user perceived QoS since the transmission of packet $m$ will have to be aborted for realtime services. On the other hand, if $R_{T M i}>R_{m}$, then $\delta_{m i}<0$ and the transmission of packet $m$ finishes before packet $m+1$ is generated, allowing other users to use the channel.

In contrast with the TA scheme, whose criterion is to maximise the throughput performance, the DSA algorithm not only considers the throughput as the selection criteria but also the delay, which represents and important parameter for many delay-sensitive multimedia services. In this sense, the DSA algorithm is intended to maximise the throughput performance while minimising the experienced delay. This design objective implies maximising $1 / \delta_{m}$ when $\delta_{m}>0$ and maximising $\left|\delta_{m}\right|$ when $\delta_{m}<0$. As a result, DSA regards a transport mode $T M_{i}$ as optimum if it maximises $\Delta_{T M i}(\gamma)$, defined as

$\Delta_{T M i}(\gamma)= \begin{cases}\frac{\Gamma_{T M i}(\gamma)}{\delta_{m i}}=\frac{\Gamma_{T M M}(\gamma) \cdot R_{T M i}}{S_{m}-D_{m} \cdot R_{T M i}}, & \delta_{m i}>0 \\ \Gamma_{T M i}(\gamma) \cdot\left|\delta_{m i}\right|=\Gamma_{T M i}(\gamma)\left(D_{m}-\frac{S_{m}}{R_{T M i}}\right), & \delta_{m i}<0\end{cases}$

or simply

$\Delta_{T M i}(\gamma)=\frac{\Gamma_{T M i}(\gamma)}{\left|\delta_{m i}\right|^{\operatorname{sgn}\left(\delta_{\mathrm{mi}}\right)}}$

with $\Gamma_{T M i}(\gamma)$ defined in expression (1) and $\operatorname{sgn}(\cdot)$ being the sign function, defined as $\operatorname{sgn}(x)=x /|x|$. Notice that maximising $\Delta_{T M i}(\gamma)$ implies maximising the throughput $\Gamma_{T M i}(\gamma)$ while minimising the delay $\delta_{m i}$. Consequently, according to the DSA criterion the optimum transport mode $T M_{\text {opt }}$ for a given channel quality $\gamma$ is selected as follows

$T M_{\text {opt }}(\gamma)=T M_{x}: \Delta_{T M x}(\gamma)=\max \left\{\Delta_{T M i}(\gamma)\right\} \quad \forall T M_{i}$

The operation of the proposed DSA algorithm can be summarised as follows. Every time a new packet has to be transmitted, the algorithm computes for each transport mode the value of $\delta_{m i}$ as defined in (5) and then evaluates expression (6) taking into account the sign of $\delta_{m i}$. Similar 
to the operation of the TA scheme, the result of each one of the previous evaluations for each transport mode is compared in order to decide the LA switching thresholds as indicated in expression (8).

The direct comparison of the TA algorithm operation with the proposed DSA algorithm reveals the necessary calculation of the LA switching thresholds for each packet to be transmitted as a possible computational drawback. However, this drawback is greatly minimised for downlink transmissions since all the calculations and decisions would be made at the base station. Moreover, as it will be shown in Sect. 5, this potential drawback is overcome by the performance improvements that can be obtained with the proposed DSA algorithm.

\subsection{Variants of the delay-sensitive algorithm}

This section proposes two variants of the DSA algorithm. The first variant, referred to as DSA-DV (Delay Variant), is intended to improve delay statistics by increasing the number of packets with $\delta_{m} \leq 0$. To this end, only transport modes that are able to guarantee that $\delta_{m i} \leq 0$ are allowed during the transmission of packet $m$, which implies that transport modes not satisfying the condition $R_{T M i} \geq R_{m}$ are removed from the set of eligible transport modes. The operation of this variant is as follows. First, the original DSA algorithm is executed. If the transport mode $T M_{\text {opt }}(\gamma)$ selected by the DSA algorithm meets the condition $R_{\text {TMopt }(\gamma)} \geq R_{m}$, then this transport mode is utilised. However, if $R_{\text {TMopt }(\gamma)}<R_{m}$, the next transport mode with the lowest $R_{T M i}$ satisfying the condition $R_{T M i} \geq R_{m}$ is used instead. In the particular case where $R_{m}>R_{T M i}$ for all available transport modes, the transport mode with the highest $R_{T M i}$ would be employed.

The second variant of the DSA algorithm, referred to as DSA-EV (Error Variant), aims at improving the quality, in terms of the error rate, for the packets transmitted with $\delta_{m} \leq 0$. To reduce such error rate, it is necessary to transmit the data with a higher error protection, which in turn implies a more frequent use of transport modes with lower $R_{T M i}$. However, expression (5) indicates that if $R_{T M i}$ is decreased, $\delta_{m i}$ increases and so does the risk of not being able to transmit the packet within the available time period $\left(\delta_{m}>0\right)$. Therefore, $R_{T M i}$ must be reduced in a controlled manner. The operation of this variant is as follow. First, the original DSA algorithm is executed. If the selected transport mode $T M_{\text {opt }}(\gamma)$ verifies $R_{T M o p t(\gamma)} \leq R_{m}$, it is employed for the following transmissions. However, if $R_{\text {TMopt }(\gamma)}>$ $R_{m}$, the transport mode with the lowest $R_{T M i}$ among all the bit-rates greater than $R_{m}$ is used instead; in this latter case, the transport mode finally selected may agree with the transport mode selected by the DSA algorithm. It is worth noting that for a given packet, the highest bit-rate allowed during the transmission is just the minimum bit-rate that enables the packet to be transmitted with $\delta_{m} \leq 0$.

\subsection{Modified error-based algorithm}

Although the EA algorithm proposed in [14] was designed to achieve a given target error rate, the results shown in [14] demonstrated that the EA performance may significantly differ from the set rate target in adaptively varying radio environments. In this context, this section proposes a modified version of the EA algorithm, which will be referred to as Modified Error-based Algorithm (MEA), to further reduce the transmission errors and effectively guarantee that the established error rate target is reached.

The proposed MEA algorithm regularly evaluates the experienced average error rate (for instance, the BLER) during previous transmissions. If the estimated average BLER is above the specific target error rate $B L E R_{\text {target }}$, the MEA algorithm will forbid, for the next transmission period, the use of the least robust transport mode out of all transport modes that could be used during the previous transmission period. On the other hand, if the estimated average BLER is below BLER $R_{\text {target }}$, the MEA algorithm will allow the use of the most robust transport mode out of all transport modes that were not permitted during the previous transmission period. If the average BLER is below $B L E R_{\text {target }}$ and all transport modes are permitted, the MEA algorithm will take no action and its operation will follow the EA principle. Also, if the average BLER is above $B L E R_{\text {target }}$ and only the use of the most robust transport mode was allowed during the previous transmission period, the MEA algorithm will maintain this situation during the next transmission period. An important design parameter for the MEA algorithm is the period over which the average BLER is estimated; this parameter is referred in the rest of this paper as averaging period.

\section{Simulation environment}

\subsection{General packet radio service}

The proposed LA algorithms have been evaluated by means of extensive system-level simulations carried out with a highly sophisticated system-level simulator based on the General Packet Radio Service (GPRS) radio interface [9]. Although a 2.5G system, GPRS has been used as a test scenario not only because of the availability of an adequate and powerful system-level evaluation platform but also because it defines various transport modes with different error correction capabilities as explained in Sect. 2.1. As a result, GPRS represents a suitable evaluation scenario for analysing the proposed LA techniques. 
To evaluate the performance of the proposed LA algorithms for delay- and error-sensitive data traffic, a real-time H.263 video traffic source has been considered in this study. The low transmission rates achieved by the GPRS radio interface may raise some questions about the feasibility of transmitting video traffic in this system. However, it is worth mentioning that the aim of this work is not to prove the feasibility of video transmissions over the GPRS technology but to analyse the performance of the proposed LA algorithms under a realistic emulation platform and compare it to that achieved with other solutions reported in the literature. To this end, the availability of a common appropriate evaluation scenario where LA can be applied suffices. Furthermore, this paper is confined to a single-slot transmission scenario. As reported in [6], link level models for multi-slot transmissions would be desirable, especially if the study of adaptive radio link techniques is considered. However, since link level models available in the literature do not contemplate a multi-slot scenario, the simulation scenario considered in this work has concentrated on single-slots transmissions. Once more, this scenario suffices to evaluate the performance improvements of the proposed LA algorithms with respect to the traditional ones.

Although the proposed LA algorithms are analysed under a GPRS-system scenario, their concept and operation are not technology-dependant and can therefore be applied to other radio interfaces.

\subsection{Simulation tool and system modelling}

The performance evaluation of a cellular system is usually conducted at two different levels: system level and link level. While the former models a mobile radio network, the later models the radio link at the bit level. Since this study is aimed at proving the benefits of new LA schemes, it concentrates on system level aspects of the operation and performance of LA algorithms. As a result, this section briefly presents the system level simulation tool that has been employed. The necessary interfaces between the link and system level studies are presented in the next section.

In order to ensure high accuracy and to account for sudden channel quality variations, this work has been conducted using an event-driven simulator working at the burst level. The simulator models a sectorised macrocellular network and concentrates on downlink transmissions. Users are assigned channels in a first-come-first-served basis and the channel is kept until all the data have been correctly transmitted. A single slot allocation strategy has been implemented by means of a random allocation scheme. Although mobility has been implemented, handover between sectors has not been considered. The main simulation parameters are summarised in Table 2. A full description of the simulation tool can be found in [9].
Table 2 Simulation parameters

\begin{tabular}{|c|c|}
\hline Parameter & Value \\
\hline Cluster size & 4 \\
\hline Cell radius & $1 \mathrm{~km}$ \\
\hline Sectorisation & $120^{\circ}$ \\
\hline Modelled interference & First and second co-channel tiers \\
\hline $\begin{array}{l}\text { Number of modelled } \\
\text { cells (wrap-around) }\end{array}$ & 25 \\
\hline Slots per sector & 16 \\
\hline Users per sector & 12 \\
\hline Traffic type & $\begin{array}{l}\text { H.263 video: } 6 \text { users/sector } \\
\text { Web browsing: } 3 \text { users/sector } \\
\text { E-mail: } 3 \text { users/sector }\end{array}$ \\
\hline Pathloss model & Okumura-Hata \\
\hline Shadowing & $\begin{array}{l}\text { Log-normal distribution }(6 \mathrm{~dB} \text { standard } \\
\text { deviation and } 20 \mathrm{~m} \text { decorrelation } \\
\text { distance })\end{array}$ \\
\hline Vehicular speed & $50 \mathrm{~km} / \mathrm{h}$ \\
\hline ARQ protocol & $\begin{array}{l}\text { Only for web browsing and e-mail users. } \\
\text { Assumed: perfect feedback of ARQ } \\
\text { report and no RLC block losses }\end{array}$ \\
\hline ARQ window size & 64 RLC blocks \\
\hline $\begin{array}{l}\text { ARQ report polling } \\
\text { period }\end{array}$ & 16 RLC blocks \\
\hline LA updating period & $60 \mathrm{~ms}$ \\
\hline
\end{tabular}

\subsection{Link-to-system level interfaces}

LA algorithms select the optimum transport mode based on the experienced channel quality conditions. As a result, the representation of such conditions is a key aspect to be considered whenever analysing the performance and operation of LA. In order to reduce the complexity and computational cost of system level simulations, the effects at the physical layer are generally included by means of Look-Up Tables (LUTs). Since the work reported in [7] demonstrated the importance of using accurate LUTs for the study of adaptive radio link techniques, a set of advanced link-to-system level interfaces working at the burst level have been considered in this study. Such interfaces were readily available for the GPRS system, which support our choice to select this system to carry out our study. These interfaces are composed of two LUTs. The interface requires as input from the system level the mean CIR experienced in a given burst. LUT-1 extracts the burst link quality for the measured burst CIR, represented by means of the BER. LUT-1 represents a Cumulative Distribution Function (CDF) of the BER for a given CIR. A random process is then used to generate the actual BER from the corresponding $\mathrm{CDF}$ (there is a BER CDF for each local mean CIR). The interest of this procedure is to model the effect of fast fading on the BER through a random 
process, thereby including the fast fading at the system level. The BER is then estimated for the four bursts used to transmit a GPRS Radio Link Control (RLC) block and LUT-2 maps the mean BER and the standard deviation of the BER over the four bursts to a corresponding BLER value. Examples of both types of interfaces can be found in [8].

\subsection{Traffic modelling}

To create a heterogeneous traffic environment two more traffic services, web browsing and email, have also been implemented. However, no channel partition has been applied between the different services.

The web browsing [1] and email traffic [11] sources have been implemented as an ON/OFF model. For both traffic models, the transmission of a new packet cannot start until the previous transmission has finished, i.e. all the data have been correctly received. The active transmission time will hence depend on the link quality conditions. Since the GPRS radio transmission rates are quite low, the implemented real-time H.263 video traffic source has a $16 \mathrm{kbit} / \mathrm{s}$ mean bit rate. The H.263 video traffic model considered [13] employs three different frame types, namely I, P and PB. Each frame type exhibits different statistical properties, which are accurately captured by the model. The video traffic model considers the following variables and properties: frame size, frame duration, correlation between frame size and frame duration, and frame transition rate. The modelling is performed at two levels. The first one determines the type of the frame to be generated. While I frames are generated at regular intervals, the generation of $\mathrm{P}$ and $\mathrm{PB}$ frames is driven by a Markov chain. Once the frame type has been decided, the second modelling level determines the frame size and duration.

\section{Performance evaluation}

To evaluate the potential benefits of the DSA and MEA LA algorithms proposed in this work, their performance is compared against that achieved with the TA and EA schemes.

\subsection{Performance measures}

The LA performance is primarily assessed in terms of throughput and delay. To this effect, it is necessary to consider not only the mean performance but also other more restrictive performance metrics, such as the minimum throughput or maximum delay experienced by $95 \%$ or even 99\% of the samples (these metrics provide a better indication of the minimum QoS provided by an algorithm).
The throughput is defined as the total number of bits successfully transmitted over the air interface divided by the radio transmission time. The delay performance is evaluated by means of the normalised delay, which corresponds to the time needed to transmit a block of data divided by the size of such block. Other interesting parameter representative of the delay performance is the number of packets transmitted before the next packet is generated, i.e. with $\delta_{m} \leq 0$. For H.263 video transmissions a packet corresponds to a H.263 video frame. The quality of the received data is evaluated by means of the BLER, which is defined as the proportion of RLC blocks successfully transmitted over the radio interface. Since a BLER below $5 \%$ would not produce a noticeable video degradation for $\mathrm{H} .263$ video transmissions [10], this value is considered as a reference threshold.

Other parameters of interest to understand the operation of the LA algorithms are the usage percentage of each CS, the proportion of RLC blocks received with the optimal $\mathrm{CS}^{2}$ and the number of $\mathrm{CS}$ changes per second requested by the LA algorithm; this last parameter is representative of the signalling load resulting from the application of LA.

\subsection{Delay-sensitive algorithm performance}

Table 3 summarises the main DSA performance results. As it can be observed, the DSA algorithm improves the mean throughput performance compared to the TA algorithm. Figure 5 highlights that the DSA improvements with respect to the traditional TA algorithm are even more important for the most restrictive QoS parameters. In fact, the results depicted in Fig. 5 show that the minimum throughput obtained for 95 and $99 \%$ of the samples is improved by 8.3 and $7.4 \%$, respectively, whereas the mean throughput improvement was equal to $2.3 \%$. As observed in Table 3 and Fig. 5, the delay variant scheme further improves the TA performance: $6.4 \%$ for the mean value, 25.9 and $54.1 \%$, respectively, for 95 and $99 \%$ of the samples. These significant improvements are due to the increase in the use of CS-4, the transport mode with the highest bit-rate, and the increase in the percentage of RLC blocks received with the optimum CS (see Table 4). The DSA and DSA-DV throughput improvements for the most poorly served users and for the average performance are not obtained at the expense of the users experiencing the highest throughput levels. In fact, Fig. 5 shows that DSA and DSA-DV improve the throughput performance for the whole range of bit-rates. In terms of the delay performance,

\footnotetext{
${ }^{2}$ A RLC block is received with the optimal CS if the used CS selected by the LA algorithm in the previous updating period is equal to the optimal one according to the instantaneous channel quality conditions experienced during the RLC block reception.
} 
Table 3 Average TA and DSA performance

\begin{tabular}{|c|c|c|c|c|c|}
\hline Parameter & TA & DSA & DSA-DV & DSA-EV & DSA-DEV \\
\hline $\begin{array}{l}\text { Throughput } \\
\text { (kbit/s) }\end{array}$ & 16.56 & 16.94 & 17.62 & 14.71 & 14.61 \\
\hline BLER (\%) & 11.18 & 12.20 & 14.11 & 6.33 & 8.20 \\
\hline $\begin{array}{l}\text { Normalised delay } \\
\quad(\mathrm{ms} / \mathrm{kbit})\end{array}$ & 55.62 & 53.21 & 48.55 & 65.26 & 63.26 \\
\hline $\begin{array}{l}\text { CS change-rate } \\
\text { (changes/s) }\end{array}$ & 4.69 & 3.94 & 2.24 & 2.74 & 2.98 \\
\hline
\end{tabular}

the results illustrated in Table 3 also demonstrate the improvement obtained by both the DSA and DSA-DV algorithms with respect to the TA algorithm. In particular, the DSA and DSA-DV algorithms reduce the mean normalised delay by 4.3 and $12.7 \%$, and the maximum normalised delay experienced by $95 \%$ of the samples by 18.8 and $32.6 \%$, respectively. These improvements are a result of a more aggressive approach in terms of the transport mode selection; as shown in Table 4, the proposed DSA and DSA-DV schemes result in a higher selection of CS-4. Table 5 indicates that the lower normalised delay obtained with the DSA and DSA-DV algorithms results in an important increase in the number of video frames that are transmitted without delay $\left(\delta_{m} \leq 0\right)$, i.e. that their transmission is finished before the next video frame is generated. Table 3 shows an increase of the mean
Table 4 TA and DSA CS selection statistics (\%)

\begin{tabular}{lcrrrl}
\hline Algorithm & CS-1 & \multicolumn{1}{c}{ CS-2 } & CS-3 & CS-4 & Optimum CS \\
\hline TA & 6.63 & 7.14 & 26.41 & 59.82 & 65.99 \\
DSA & 3.37 & 2.92 & 27.98 & 65.73 & 71.17 \\
DSA-DV & 0.00 & 0.65 & 14.00 & 85.34 & 85.77 \\
DSA-EV & 2.60 & 39.54 & 38.02 & 19.84 & 80.14 \\
DSA-DEV & 1.48 & 39.12 & 34.43 & 24.97 & 80.39 \\
\hline
\end{tabular}

BLER experienced with the DSA and DSA-DV algorithms with respect to that observed for the TA algorithm. Such increase is due to a higher utilisation of the least robust transport mode. Although the throughput and delay performance improvements achieved with the DSA and DSADV algorithms are obtained at the expense of a higher mean BLER, the results shown in Table 5 demonstrate that a higher percentage of video frames transmitted without delay $\left(\delta_{m} \leq 0\right)$ and with the required 5\% BLER target suggested by Hanzo et al. [10] are still attainable with both the DSA and DSA-DV algorithms.

On the other hand, Table 3 shows that the DSA-EV algorithm, which was originally designed to enhance the error performance of the DSA algorithm, is able to reduce the experienced mean BLER. The DSA-EV scheme is able to achieve an average BLER reduction of 4.85 and $5.87 \%$, respectively, with respect to the TA and DSA schemes. Although such BLER improvements are achieved at the
Fig. 5 Cumulative distribution function of H.263 throughput performance

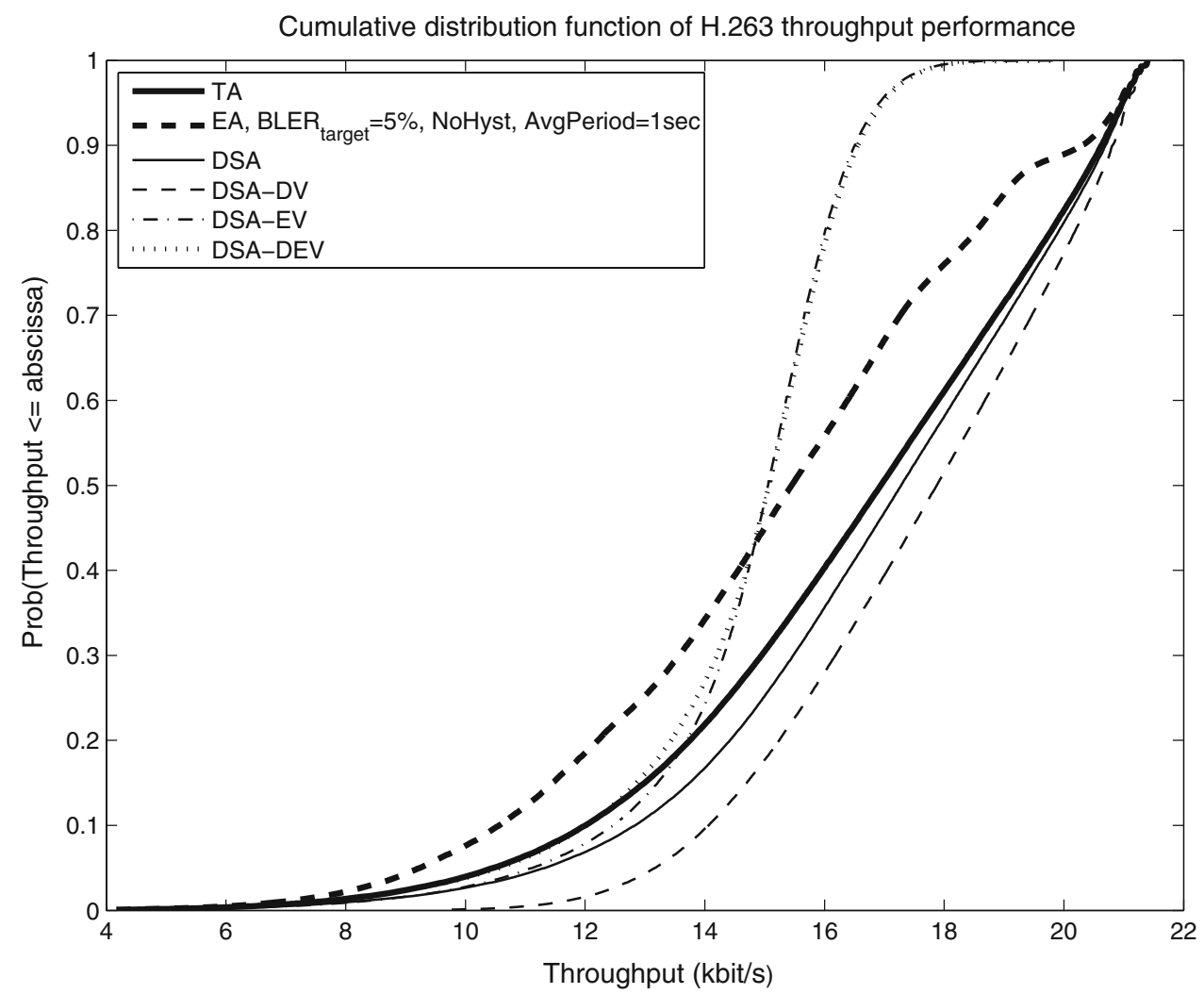


Table 5 TA and DSA video frames transmission statistics

\begin{tabular}{llllll}
\hline Parameter & TA & DSA & DSA-DV & DSA-EV & DSA-DEV \\
\hline Percentage of frames with $\delta_{m} \leq 0$ & 69.16 & 73.92 & 82.65 & 65.91 & 72.85 \\
$\begin{array}{l}\text { Percentage of frames with } \delta_{m} \leq 0 \\
\quad 44.98\end{array}$ & 46.45 & 49.02 & 55.01 & 57.21 \\
$\quad$ and BLER $\leq 5 \%$ & & & & \\
\hline
\end{tabular}

expense of a higher transmission delay and lower percentage of video frames with $\delta_{m} \leq 0$, the DSA-EV proposal is able to considerably increase the percentage of video frames transmitted with $\delta_{m} \leq 0$ and with BLER $\leq 5 \%$ (see Table 5). The DSA-EV achievements are due to an important decrease in the use of CS-4 and a more frequent use of transport modes with higher error protection, in particular CS-2 and CS-3 (see Table 4). The DSA-EV error performance improvements are achieved at the expense of reducing the throughput performance with respect to the DSA scheme (Table 3). In fact, Fig. 5 shows that the maximum throughput achieved is slightly lower for the DSA-EV algorithm than for the TA and DSA algorithms. However, Fig. 5 also shows that DSA-EV can actually improve the TA throughput performance due to the use of more robust transport modes under unfavourable channel quality conditions. Although the DSA-EV algorithm usually employs CSs with lower bit-rates, the higher protection of these CSs can actually lead to higher throughput under poor channel quality conditions. On the other hand, while the TA criterion can result in higher throughput values under good channel quality conditions due to the use of higher bit-rate CSs with lower error protection, such transport mode selection approach also results in lower throughput performance under bad channel quality conditions.

As previously mentioned, a drawback of the DSA-EV variant is its poorer delay performance (see Tables 3 and 5). This possibility was pointed out in Sect. 3.2 as a potential risk if the transmission bit-rate is excessively decreased. To correct this problem, a combination of the DSA-DV and DSA-EV schemes has been analysed, which is referred to as DSA-DEV (Delay and Error Variant). The DSA-DEV proposal executes the DSA-EV algorithm during the transmission of a video frame, except for the last LA updating period where the DSA-DV algorithm is executed instead. The obtained results demonstrate that the DSA-DEV algorithm also achieves a significant mean BLER reduction with respect to the initial DSA algorithm (see Table 3), while approximately maintaining the percentage of video frames transmitted before the next one is generated, i.e. with $\delta_{m} \leq 0$ (see Table 5). Moreover, the percentage of video frames transmitted simultaneously with $\delta_{m} \leq 0$ and BLER $\leq 5 \%$ is improved with respect to the initial DSA algorithm (10.76\%), and even with respect to the DSA-EV algorithm (2.2\%).
Another indication of the better operation of the DSA proposals with respect to TA is the lower signalling load they generate. While the TA algorithm results in $4.69 \mathrm{CS}$ changes per second, the DSA algorithm only requests 3.94 CS changes per second (see Table 3 ). This figure not only demonstrates that the mode selection of the proposed algorithm is more accurate but also that the signalling load associated with the use of LA is considerably reduced (16\%) with the DSA algorithm. Further improvements are observed for the variants of the DSA algorithm (up to $52 \%$ for the DSA-DV algorithm).

This section has concentrated on the DSA performance for H.263 video services. However, the web browsing and email services performance has also been analysed as part of this study. Although their performance is not described in detail due to the lack of space, it is worth highlighting that using the DSA algorithms for H.263 video transmissions has not degraded the web and email performance.

\subsection{Modified error-based algorithm performance}

Table 6 compares the performance obtained with the TA and EA algorithms. While the TA algorithm obtains a higher throughput performance, the EA algorithm improves the experienced BLER. However, it can be observed that the difference between both algorithms is not very significant, and more importantly, that the EA average BLER is well above the desired target. This pattern was also observed in [14], although the difference between the measured BLER and the target error rate was smaller. In fact, while this difference is above $100 \%$ for the results reported in Table 6 , the difference was equal to $53 \%$ in [14]. The higher difference obtained in this work can be due to the fact that while [14] was conducted using a simple LUT, this work is based on more sophisticated link-to-system level interfaces. At this point, it is important to note that [8] has already demonstrated the effect that link-to-system

Table 6 TA and EA performance

\begin{tabular}{lrr}
\hline Parameter & TA & EA \\
\hline Mean throughput (kbit/s) & 16.56 & 16.24 \\
Minimum throughput for 95\% of samples (kbit/s) & 10.46 & 9.92 \\
Average BLER (\%) & 11.18 & 10.60 \\
Percentage of frames with BLER $\leq 5 \%$ & 60.75 & 61.10 \\
\hline
\end{tabular}


Table 7 Performance with fixed coding schemes

\begin{tabular}{lrrrr}
\hline Parameter & CS-1 & CS-2 & CS-3 & CS-4 \\
\hline Average BLER (\%) & 1.08 & 2.54 & 4.25 & 16.93 \\
$\begin{array}{c}\text { Percentage of frames } \\
\text { with BLER } \leq 5 \%\end{array}$ & 96.42 & 92.12 & 87.08 & 59.13 \\
\hline
\end{tabular}

level interfaces have on the predicted performance and operation of LA.

To better understand the MEA performance and the need to implement MEA, it is interesting to analyse the results obtained if no LA is used, i.e. a fixed CS is employed during the entire simulation. Such performance, illustrated in Table 7, is useful to explain why the EA algorithm does not perform as expected. As it can be observed from this table, the coding scheme that provides the higher BLER and the lower percentage of video frames transmitted with a BLER lower than 5\% is CS-4. In fact, all other coding schemes offer an average BLER that satisfies the target error rate for H.263 video transmissions. Since CS-4 was the most used coding scheme when considering the application of the EA algorithm (its usage percentage was equal to $57.7 \%$ ), it can be concluded that the use of CS-4 is at the origin of the EA poor BLER performance.

Taking into account the results shown in Table 7, a simple LA algorithm to achieve a low target error rate simply consists in forbidding the use of the least robust coding schemes. The performance obtained with a LA algorithm that forbids the use of CS-4 and only considers CS-1, CS-2 and CS-3 in its adaptation process is illustrated in Table 8. As it can be observed from this table, the proposed algorithm obtains an average BLER below 5\% and transmits the majority of video frames with a BLER below 5\%. This improvement in terms of BLER performance is due to an important decrease in the proportion of wrong side failures (a wrong side failure corresponds to the case where a user is using a non-optimal CS that is not robust enough for correct reception). In fact, while this proportion was equal to $10.65 \%$ in the case of the EA algorithm, it is equal to $5.43 \%$ with the proposed simple

Table 8 Proposed simple LA algorithms

\begin{tabular}{lll}
\hline Parameter & $\begin{array}{l}\text { Without } \\
\text { CS-4 }\end{array}$ & $\begin{array}{l}\text { Without CS-4 } \\
\text { and CS-3 }\end{array}$ \\
\hline Mean throughput (kbit/s) & 14.30 & 12.92 \\
$\begin{array}{l}\text { Minimum throughput } \\
\quad \text { for 95\% of the samples } \\
\quad \text { kbit/s) }\end{array}$ & 10.29 & 10.63 \\
Average BLER (\%) & & \\
$\begin{array}{l}\text { Percentage of frames with } \\
\text { BLER } \leq 5 \%\end{array}$ & 8.57 & 2.19 \\
\hline
\end{tabular}

LA algorithm. As illustrated in Table 8, the BLER performance achieved with the proposed simple LA algorithm is obtained at the cost of an important reduction of the throughput and of the proportion of RLC blocks received with the optimal CS. While this proportion was equal to $64.2 \%$ with the EA algorithm, it is equal to $14.2 \%$ for this simple proposal. However, the proposed algorithm also reduces the signalling load associated with the use of LA, which can be estimated by means of the average number of CS changes per second. While this number was equal to 5.02 with the EA algorithm, it has decreased to 1.96 with the simple LA algorithm. In fact, this reduction in the average number of CS changes per second shows that a high proportion of the signalling load associated with the use of LA is due to the use of the least robust coding scheme (CS-4). As it can be observed from Table 8, it would be possible to further improve the BLER performance if CS-3 was also forbidden in the LA process. Once more this improvement in terms of BLER would be obtained at the cost of a poorer throughput performance.

Although the previous simple proposals are able to reduce the experienced BLER value below the desired target, it is worth mentioning that the observed proportion of right-side failures (a right-side failure corresponds to the case where a user is using a non-optimal CS but one robust enough for correct reception) was too high. In particular, $80.41 \%$ when CS-4 is forbidden and $91.69 \%$ when both CS-4 and CS-3 are forbidden. These values reveal the inefficient operation of the simple LA algorithms described since an important number of RLC blocks were not transmitted using the optimal CS, which results in a lower throughput and delay performance than that necessary to maintain the target error rate. This observation suggests the possibility of a less restrictive, more elaborated approach in order to control the experienced error rate. In this context, the MEA algorithm was proposed.

The performance achieved with the MEA algorithm for different averaging periods is illustrated in Tables 9 and 10. Table 9 shows that the proposed algorithm allows to trade-off between throughput and error rate performances by simply changing the averaging period. In fact, the results obtained show that the BLER can be decreased, until a certain level, by increasing the averaging period. As shown in Table 10, increasing the averaging period reduces the use of CS-4 and the proportion of wrong side failures, and increases the proportion of right side failures. While the use of CS-4 and an important proportion of wrong side failures are generally at the origin of transmission errors, a high proportion of right side failures indicates that the proposed algorithm tends to protect, sometimes in excess, the transmissions from radio propagation errors. However, this effect is not maintained if the averaging period is increased in excess (i.e. when considering an averaging 
Table 9 MEA performance as a function of the averaging period

\begin{tabular}{lrrrr}
\hline Parameter & \multicolumn{1}{c}{$1 \mathrm{~s}$} & $12 \mathrm{~s}$ & $18 \mathrm{~s}$ & $36 \mathrm{~s}$ \\
\hline Mean throughput (kbit/s) & 15.31 & 15.02 & 14.79 & 14.41 \\
Minimum throughput for 95\% of the & 9.24 & 9.04 & 9.04 & 9.30 \\
$\quad$ samples (kbit/s) & & & & \\
Average BLER (\%) & 6.35 & 5.65 & 5.61 & 6.13 \\
Percentage of frames with BLER $\leq 5 \%$ & 75.10 & 77.87 & 78.11 & 76.70 \\
\hline
\end{tabular}

Table 10 MEA CS selection statistics

\begin{tabular}{lrrrr}
\hline Parameter & \multicolumn{1}{c}{$1 \mathrm{~s}$} & $12 \mathrm{~s}$ & $18 \mathrm{~s}$ & $36 \mathrm{~s}$ \\
\hline Usage percentage of CS-1 & 14.23 & 13.07 & 12.93 & 10.94 \\
Usage percentage of CS-2 & 16.09 & 22.49 & 24.48 & 26.50 \\
Usage percentage of CS-3 & 35.65 & 36.54 & 38.19 & 45.12 \\
Usage percentage of CS-4 & 34.03 & 27.90 & 24.40 & 17.44 \\
Optimal CS (\%) & 43.38 & 37.03 & 33.70 & 27.81 \\
Wrong side failures (\%) & 6.41 & 5.99 & 5.96 & 6.47 \\
Right side failures (\%) & 50.21 & 56.99 & 60.34 & 65.72 \\
\hline
\end{tabular}

period of $36 \mathrm{~s})$. As the results presented in Table 10 demonstrate, using an averaging period of $36 \mathrm{~s}$ provides a higher proportion of wrong side failures than using any of the other averaging periods. This increase is not due to a higher usage of CS-4 but to an important increase in the usage percentage of CS-3, a coding scheme with a low error protection, and a decrease in the usage percentage of the most robust coding scheme (CS-1).

It is possible to further reduce the experienced BLER by considering the use of hysteresis thresholds. In this case, the LA algorithm operates in the same way as when not considering hysteresis thresholds if the average BLER measured during the previous averaging period is higher than 5\%. On the other hand, the algorithm allows the use of the most robust CS out of all CS that were previously forbidden, only if the averaged BLER goes below 4 and not $5 \%$ (hysteresis margin). No action is performed if the averaged BLER is between 4 and 5\%. The operation of the proposed algorithm has also been evaluated with hysteresis thresholds equal to a BLER of 4 and $4.5 \%$. In this case, the performance will be shown for an averaging period of $14 \mathrm{~s}$ and not 18 since a lower BLER (5.22\%) was measured with the $14 \mathrm{~s}$ period. As shown in Fig. 6, the use of hysteresis thresholds improves the BLER performance and increases
Fig. 6 Percentage of H.263 video frames transmitted with BLER $\leq 5 \%$ for the MEA proposal

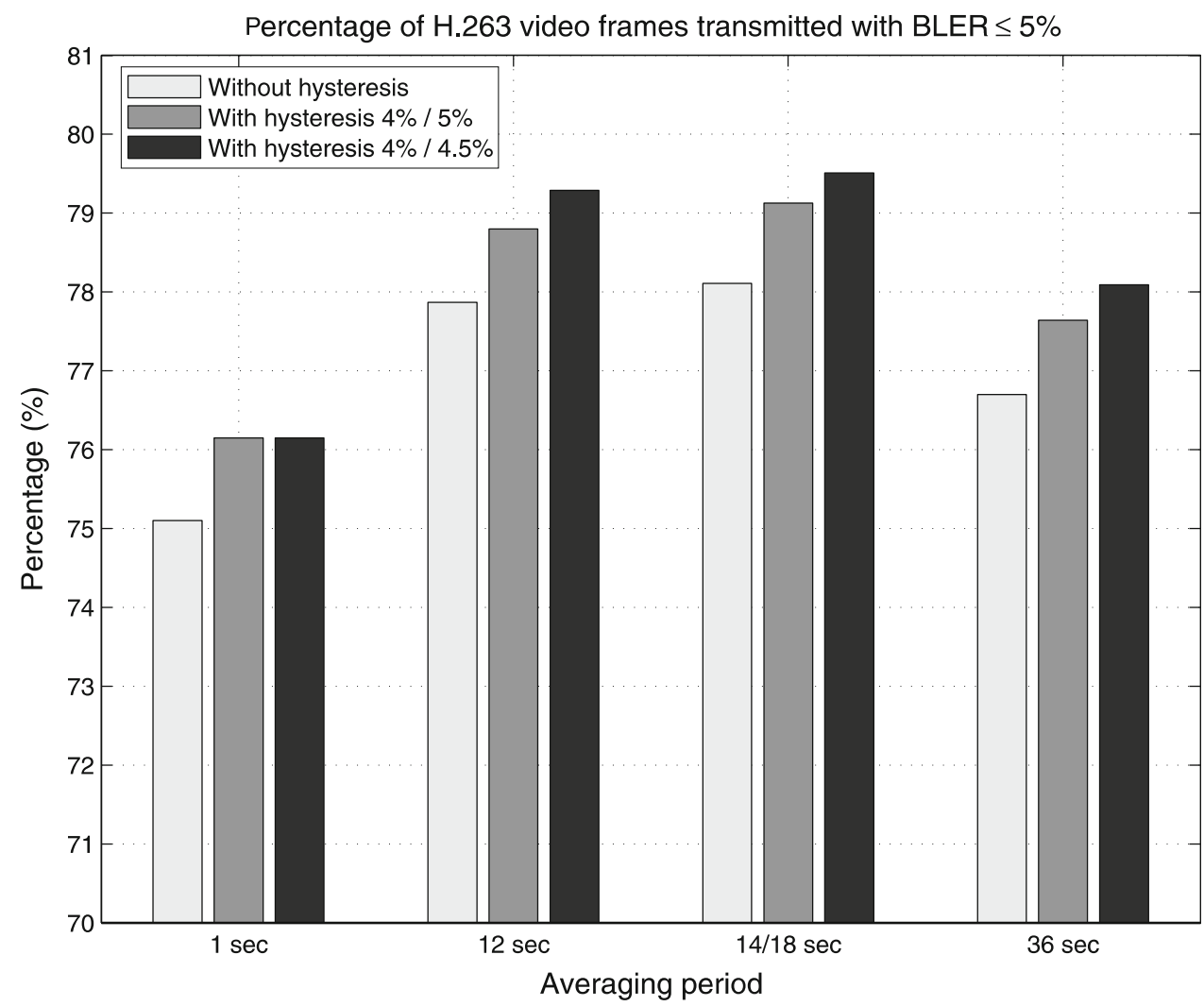


the percentage of video frames transmitted with a BLER below 5\%. Figure 6 also demonstrates that the best BLER performance is obtained with the most restrictive hysteresis thresholds; the $4-4.5 \%$ hysteresis margin allows for high error performance improvements with respect to the EA algorithm. In particular, the use of MEA together with the 4-4.5\% hysteresis threshold is able to reduce the mean BLER from 10.60 to $5.22 \%$. The percentage of video frames transmitted with a BLER lower than $5 \%$ is increased by $18.4 \%$. Moreover, while the EA algorithm results in 5.02 CS changes per second, the MEA algorithm only requests $2.30 \mathrm{CS}$ changes per second, which implies a reduction equal to $54.2 \%$ in the signalling load associated to the use of LA. To conclude, the obtained results have demonstrated that the objective set by the EA algorithm can be more effectively fulfilled with the proposed MEA scheme, which not only improves the error performance but also reduces the signalling load associated to the use of LA.

\section{Conclusions}

This paper has proposed new Link Adaptation algorithms designed to improve the performance of services with tight delay and error constraints. The algorithms have been evaluated in a dynamic radio environment and their performance has been compared to the commonly accepted and widely used throughput-based and error-based algorithms. The obtained results demonstrate that significant improvements in terms of throughput, transmission delay, error performance and signalling load can be obtained with the proposed schemes.

Acknowledgements This work has been supported by the Ministry of Education and Science (Spain) and FEDER funds under the project TEC2005-08211-C02-02 and by the Generalitat Valenciana under the projects GV05/189 and ACOMP07/256.

\section{References}

1. Barford, P., \& Crovella, M. (1998). Generating representative web workloads for network and server performance evaluation. In Proceedings of the ACM SIGMETRICS Joint International Conference and Measurement and Modeling of Computer Systems, June 1998 (pp. 151-160).

2. Dunlop, J., Cosimini, P., Graham, G., \& Le-Strat, E. (1994). Estimation of the performance of an adaptive air interface in mobile radio. In Proceedings of the RACE Mobile Telecommunications Workshop, May 1994 (pp. 47-51).

3. ETSI-SMG. (1997). EDGE feasibility study, Work item 184; Improved data rates through optimized modulation. Tdoc 97-331 (December).

4. Fabri, S., Cellatoglu, A., \& Kondoz, A. (1999). Transmission of multimedia services over GPRS using MPEG-4 coded video. In
Proceedings of the IEEE Vehicular Technology Conference, September 1999 (pp. 401-405).

5. Furuskär, A., Mazur, S., Müller, F., \& Olofsson, H. (1999). EDGE: Enhanced data rates for GSM and TDMA/136 evolution. IEEE Personal Communications, 6(3), 56-66. doi:10.1109/98. 772978.

6. Gozalvez, J., \& Dunlop, J. (2000). On the effect of correlation in multislot link layer analysis for GPRS. In Proceedings of the IEEE Vehicular Technology Conference, September 2000 (pp. 444-450).

7. Gozalvez, J., \& Dunlop, J. (2003). On the importance of using appropriate link-to-system level interfaces for the study of link adaptation. In Proceedings of the IST Mobile \& Wireless Communications Summit, June 2003 (pp. 441-445).

8. Gozalvez, J., \& Dunlop, J. (2004). Link level modelling techniques for analysing the configuration of link adaptation algorithms in mobile radio networks. In Proceedings of European Wireless, February 2004 (pp. 325-330).

9. Gozalvez, J., \& Dunlop, J. (2005). System performance and adaptive configuration of link adaptation techniques in packetswitched cellular radio networks. The International Journal of Computer and Telecommunications Networking, 49(3), 404-426.

10. Hanzo, L., Cherriman, P., \& Streit, J. (2001). Wireless video communications: Second to third generation systems and beyond. NY: IEEE Press.

11. Ho, J., Zhu, Y., \& Madhavapeddy, S. (1999). Throughput and buffer analysis for GSM general packet radio service (GPRS). In Proceedings of the IEEE Wireless Communications and Networking Conference, September 1999 (pp. 1427-1431).

12. Kodikara, C., Fabri, S. N., \& Kondoz, A. M. (2002). Performance improvement for real-time video communications by link adaptation in E-GPRS networks. In Proceedings of the Third International Conference on $3 G$ Mobile Communication Technologies, May 2002 (pp. 489-494).

13. Lazaro, O., Girma, D., \& Dunlop, J. (2004). H.263 video traffic modelling for low bit rate communications. In Proceedings of the IEEE Personal, Indoor and Mobile Radio Communications Conference, September 2004 (pp. 2124-2128).

14. Leung, K. K., Driessen, P. F., Chawla, K., \& Qiu, X. (2001). Link adaptation and power control for streaming services in EGPRS wireless networks. IEEE Journal on Selected Areas in Communications, 19(10), 2029-2039. doi:10.1109/49.957316.

15. Luo, W., Balachandran, K., Nanda, S., \& Chang, K. (2000). Packet size dependent link adaptation for wireless packet data. In Proceedings of IEEE Globecom, November 2000 (pp. 53-56).

16. Nakamura, M., Awad, Y., \& Vadgama, S. (2002). Adaptive control of link adaptation for high speed downlink packet access (HSDPA) in W-CDMA. In Proceedings of the International Symposium on Wireless Personal Multimedia Communications, October 2002 (pp. 382-386).

17. Parkvall, S., Dahlman, E., Frenger, P., Beming, P., \& Persson, M. (2001). The evolution of WCDMA towards higher speed downlink packet data access. In Proceedings of the IEEE Vehicular Technology Conference, May 2001 (pp. 2287-2291).

18. Queseth, O., Gessler, F., \& Frodigh, M. (1999). Algorithms for link adaptation in GPRS. In Proceedings of the IEEE Vehicular Technology Conference, May 1999 (pp. 943-947).

19. Quiao, D., Choi, S., \& Shin, K. G. (2002). Goodput analysis and link adaptation for IEEE 802.11a wireless LANs. IEEE Transactions on Mobile Computing, 1(4), 278-292. doi:10.1109/ TMC.2002.1175541.

20. Ramachandran, S., Bostian, C. W., \& Midkiff, S. F. (2005). A link adaptation algorithm for IEEE 802.16. In Proceedings of the IEEE Wireless Communications and Networking Conference, March 2005 (pp. 1466-1471). 


\section{Author Biographies}

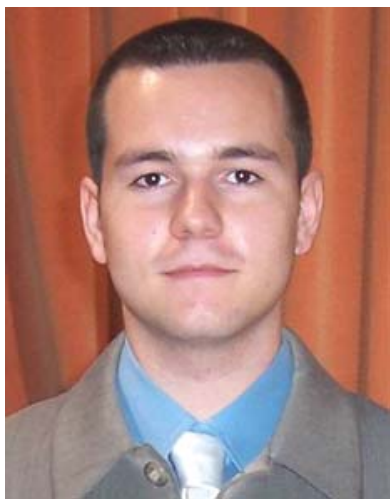

Miguel López-Benítez received a Telecommunication Engineering degree (first-class honors) in 2006 from University Miguel Hernández (UMH), Elche, Spain, and was the recipient of the 2006 University Education National Award, a distinction from the Spanish Ministry of Education and Science to the best national academic records. His degree thesis was awarded a national research prize from the France Telecom Foundation in the context of the 5th Archimedes University Competition, the main young scientist contest in Spain organized by the Spanish Ministry of Education and Science. From February 2003 to July 2006 he actively participated in several research activities in the Signal Theory and Communications Division of the University Miguel Hernández. Since September 2006 he is with the Radio Communication Group at the Department of Signal Theory and Communications of the Technical University of Catalonia (Universitat Politècnica de Catalunya, UPC), Barcelona, Spain, where he currently is pursuing a $\mathrm{Ph} . \mathrm{D}$. degree in Telecommunication Engineering with the support of a FPU grant from the Spanish Ministry of Education and Science. He has been actively involved in European-funded projects AROMA and NEW$\mathrm{COM}++$ along with Spanish project COGNOS. His current research interests include the field of mobile radio communication systems, with a special emphasis on radio resource management, heterogeneous wireless systems, quality of service provisioning, and opportunistic/dynamic spectrum access in cognitive radio networks.

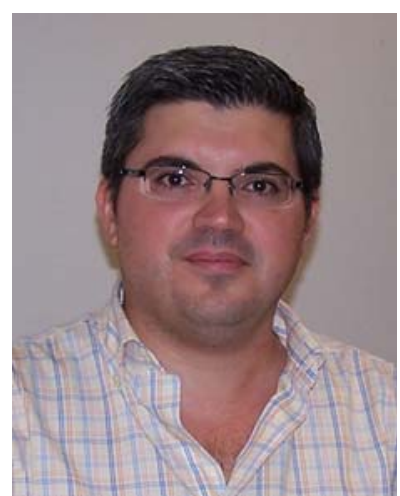

Javier Gozálvez received an Electronics Engineering degree from ENSEIRB (Bordeaux, France), a DEA in Electronics from Université de Bordeaux I and a Ph.D. in Mobile Communications from the University of Strathclyde (Glasgow, UK). Since October 2002, he is at the Signal Theory and Communications Division of the University Miguel Hernández (Elche, Spain) where he received the 2005 University Miguel Hernandez Young Researcher Award. He is currently an Associate Professor and Director of the Uwicore research laboratory (www.uwicore.umh.es), leading mobile and wireless communications research activities in various European and national research projects. He serves as Mobile Radio Senior Editor of the IEEE Vehicular Technology Magazine and as Associate Editor of the IEEE Communication Letters journal. He is the Technical Program co-Chair for the 69th IEEE Vehicular Technology Conference Spring 2009, was the General co-Chair for the 3rd International Symposium on Wireless Communications Systems (ISWCS'2006), Speakers Chair for the IEEE Vehicular Technology Conferences $(2004,2005,2007)$ and General co-Chair, and founder, of the 2007 and 2008 IEEE International Symposium on Wireless Vehicular Communications (WiVeC). His current research interests include: radio resource management, heterogeneous systems, wireless vehicular communications and wireless sensor networking. 